\title{
Dialogue in the Wild: \\ Learning from a Deployed Role-Playing Game with Humans and Bots
}

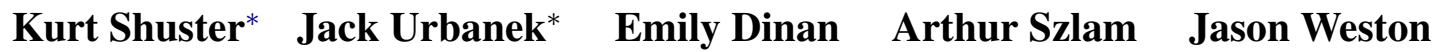

Facebook AI Research

\begin{abstract}
Much of NLP research has focused on crowdsourced static datasets and the supervised learning paradigm of training once and then evaluating test performance. As argued in de Vries et al. (2020), crowdsourced data has the issues of lack of naturalness and relevance to real-world use cases, while the static dataset paradigm does not allow for a model to learn from its experiences of using language (Silver et al., 2013). In contrast, one might hope for machine learning systems that become more useful as they interact with people. In this work, we build and deploy a role-playing game, whereby human players converse with learning agents situated in an open-domain fantasy world. We show that by training models on the conversations they have with humans in the game the models progressively improve, as measured by automatic metrics and online engagement scores. This learning is shown to be more efficient than crowdsourced data when applied to conversations with real users, as well as being far cheaper to collect.
\end{abstract}

\section{Introduction}

Humans learn to use language over the course of their lives from the interactions they have with the world and other people. Yet, the prevailing dominant paradigm in natural language processing (NLP) research is to build a fixed dataset from which to train a model and then freeze it, without any ability for the model to interact with humans using language at training time at all. While we need such interaction in order to study human-machine communication to its full extent, constraints usually inhibit such research. Firstly, conducting such experiments can be costly. Many datasets in NLP are collected with crowdsourcing, whereby one pays the crowdworkers to perform interaction and

\footnotetext{
* Equal Contribution
}

annotation tasks. This leads to several issues, not least that research budgets for paying crowdworkers mean that data will have a limit. Secondly, as crowdworkers are motivated by pay, not by interest in the tasks themselves, the data distribution may not match the desired one (de Vries et al., 2020).

In this work we study the ability of dialogue agents in an open world ${ }^{1}$ to iteratively learn from conversations with intrinsically motivated humans. In order to engage humans at scale, we build and deploy a (free to play) game with a purpose (Von Ahn, 2006) whereby human players role-play characters and converse with other characters (that are our learning models) situated within the game world. We choose a rich fantasy game world, in order to maximize engagement. Our system iterates between collecting data of human-model interactions, retraining updated models on the newly collected data, and redeploying them. Simultaneously, it provides a natural metric to evaluate and compare models online using human continue rates (that is, how long human players continue playing).

We show that we can successfully collect, retrain and redeploy models, starting from a crowdsourced base model, that improve both offline automatic metrics and human continue rates. Our overall system is then engaging enough that we can collect data at a rate that is $1 / 5^{t h}$ of the price per utterance of crowdsourcing, where the cost of our method is the cost of advertisements that make players aware of the game. Moreover, the data we collect is also more effective per utterance at improving continue rates due to being more on-distribution than crowdsourced data. As our models improve, these rates improve as well, as the continuation rate increases

\footnotetext{
${ }^{1}$ In this work we study dialogue that can be about any topic but within the scope of a fantasy game world. Note this differs from open-domain dialogue talking about our world, e.g. the game players can talk about the sauce recipe from Bredwell across the sea (see Fig. 1), but not about the pizza in Chicago.
} 
- meaning relatively more data is collected. Overall, our work provides good evidence that lifelong dialogue learning in deployed systems with intrinsically motivated humans (rather than crowdworkers) can be successful, in particular by embedding such learning within games.

The training code and parameters of the models deployed, and the data collected in this work will be made publicly available for reproducibility and further research by the community.

\section{Related Work}

Open-Domain Dialogue Dialogue in the opendomain (chitchat) setting, which involves chat about any topic, rather than a specific goal-directed topic, is commonly studied in the train/valid/test static dataset paradigm utilizing supervised learning. A number of crowdsourced or scraped datasets have been developed to that end, including Daily Dialogue (Li et al., 2017), PersonaChat (Zhang et al., 2018), Empathetic Dialogues (Rashkin et al., 2019) and Wizard of Wikipedia (Dinan et al., 2019b); see Huang et al. (2020) for a review.

LIGHT In this work we specifically focus on dialogue setting of LIGHT (Urbanek et al., 2019). LIGHT focuses on situated characters playing character roles that can chat about any topic, within the context of a medieval fantasy world. This setting is known to be engaging for human role-players, and also alleviates some safety concerns in that the roleplaying means they should not divulge personally identifying information. The authors crowdsourced a dialogue dataset consisting of $8.5 \mathrm{k}$ episodes and $111 \mathrm{k}$ utterances, which they publicly released. We refer to this as LIGHT MTurk data, or LIGHT data for short, in the rest of this paper. In this work we utilize this data to build a deployed system whereby players can converse with models, and we can study lifelong learning with these models using the information in these new conversations.

Lifelong Learning Lifelong learning is a machine learning paradigm whereby deployed models can interact with the world and iteratively improve themselves from the things they learn, eschewing the standard approach of a fixed training set from which a model is trained once (Silver et al., 2013). We note there are other closely related concepts to the topics in this work, such as incremental learning (Castro et al., 2018), continual reinforcement learning (Ring, 1994) and never-ending learning
(Carlson et al., 2010; Mitchell et al., 2018).

Interactive Dialogue Learning Learning from dialogue interaction is common in reinforcement learning settings, where the feedback is a scalar rather than solely the dialogue messages themselves (Levin et al., 2000; Schatzmann et al., 2006; Rieser and Lemon, 2011; Liu and Lane, 2017; Serban et al., 2017), which is most common in a goaloriented setting where completion of the goal can provide such rewards, but not so in chitchat settings (Roller et al., 2020), which do not have rewards.

Closer to our work, is the self-feeding chatbot (Hancock et al., 2019), where dialogue models were used to collect data to improve themselves via crowdsourcing utilizing the PersonaChat task. Related approaches have also been applied to the cases of question answering (Li et al., 2016a,b), and in simulators (Mazumder et al., 2019; Nguyen and Daumé III, 2019) as well. Liu et al. (2018) applied such an approach to goal-oriented tasks. Our work differs from these works in that we study a deployed user-facing system in an open-ended game environment, rather than more limited data from paid crowdworkers. For other related works see Padmakumar and Mooney (2020).

Deployed Dialogue Systems While there are a number of deployed virtual assistants, many of these products are not ideal platforms for the research community. Their proprietary nature and commercial importance, coupled with privacy concerns, means they are neither accessible to researchers, nor amenable to public reproducible research. A near-exception is the Alexa challenge (Ram et al., 2018) which allows university-based researchers access to a commercial user-base for the span of the competition, however, the data and models are also not released to the rest of the research community, unlike in our work.

\section{Dialogue as a role-playing game}

In this section we describe the game that we will build and deploy, which is a role-playing game, designed to both train and evaluate dialogue agents.

Core Mini-Game The core game involves pairing two agents in a given setting - where one is a human player and the other is a dialogue agent with an underlying machine learning model. The two players are assigned characters, with given names and backstories (personas), and their current location and its description. See Figure 1 for examples. 

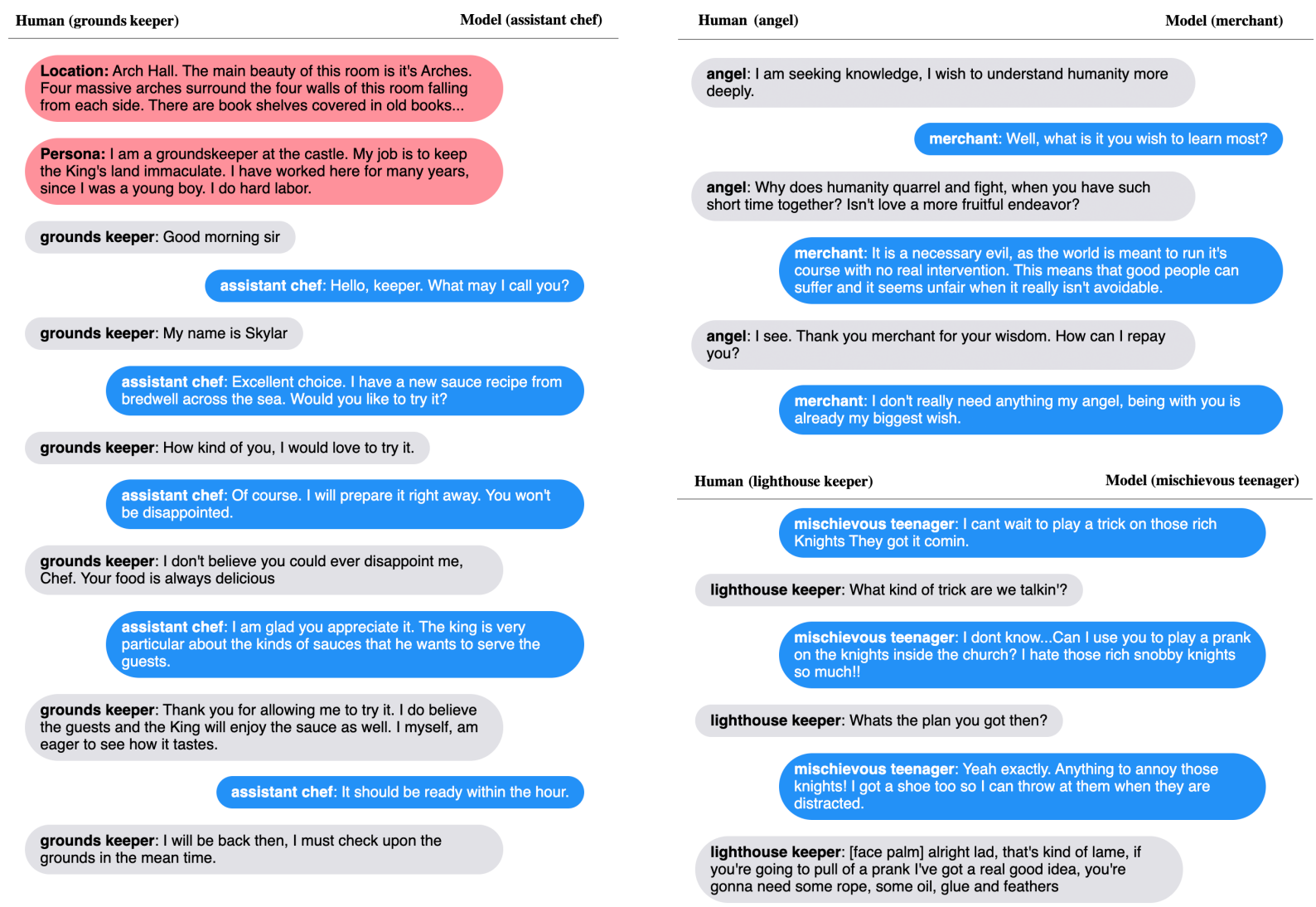

Figure 1: Example collected dialogues from humans role-playing in our deployed system, conversing with models. (Left) a conversation complete with described location and player persona; (Right) excerpts from two other conversations (out of 41,131 collected) to demonstrate the diversity of the dialogue in the fantasy game world.

Each player's goal is to act out (role-play) their character's dialogue in the given situation. We refer to one such dialogue episode as a mini-game. Dialogue in the game is in English.

Role-Playing (Acting) Score We take advantage that role-playing is a pursuit that a large number of human players find fun (Horsfall and Oikonomou, 2011), and are hence naturally engaged in the openended nature of this process. However, to encourage and further motivate players to play their characters well, we introduce the concept of an (automated) dungeon master (DM), who will assess the quality of the player's role-playing. For each dialogue turn, we apply a learned model to the human player's dialogue, which assesses how likely their utterance is given the context. We convert this to a score, between 1 and 5 stars, that is presented to the human player, to reward them for good acting. While this signal is noisy, because our DM model is not perfect, it gives motivating feedback to the players to continue playing.
Game Loop Each dialogue (mini-game) consists of 6 turns of dialogue per agent (12 total). At the end of the mini-game the human player is presented with four choices: (i) choose to move to a new location, where they will continue to play this character, but meet a new character to converse with; (ii) stay in the same room but wait for a new character to arrive to converse with; (iii) change to role-play a completely new pair of characters in a new setting; or (iv) end the game. These choices encourage the player to choose another mini-game that they are most interested in, and the variety of mini-games gives different role-playing possibilities, making the dialogue data more diverse. Further details of the game are described in Appendix A.

Deployment and Advertising We deployed the game using Facebook Messenger on CPU-only servers, and used the publicly-available ad flow to advertise on Facebook. The target audience for our ads were people with interests in online gaming, role playing, and role playing games in particular. We also used lookalike audiences to those who engaged with the game. We ran ads like that shown 
in Figure 4 with a call-to-action for people to try out the game.

\section{Lifelong Dialogue Learning}

\subsection{Models}

Retrieval Models All the models we have currently deployed are retrieval models for safety reasons as we can vet each possible response by filtering the train set candidates (see Appendix A). In particular, we use the Poly-Encoder (PE) Transformer architecture as a base (Humeau et al., 2019), as it provides state of the art results compared to other retrieval models, whilst being tractable to deploy. PE encodes the context with a standard bidirectional transformer, but produces an encoding into a fixed small number of codes, $N$. We tried values of $N=5$ and $N=20$. Each label candidate then attends to these codes before producing a final matching score. The model is trained with crossentropy given the correct label, and by subsampling negative examples from the given training batch.

Architecture and Training Choices We employ the $90 \mathrm{M}$ and $622 \mathrm{M}$ parameter models from (Roller et al., 2020) that have been pre-trained on 1.5B training examples from pushshift.io Reddit, which we then fine-tune. We also consider two other enhancements, chosen to mitigate problems that we observed with the models: (i) negative context training, whereby negatives are also selected from the immediate dialogue history as well as the batch which can help reduce a model's tendency to repeat itself (Holtzman et al., 2019; Welleck et al., 2020); and (ii) decoding control (See et al., 2019) whereby at decoding time responses are rescaled before scoring based on their specificity (normalized inverse document frequency). The latter can control the genericness of the responses, which is known to affect human judgments.

Generative Models In addition to the deployed models, we also train and evaluate generative models offline, where safety concerns are less important as the models are not user-facing. We employ an encoder-decoder Transformer architecture using the state of the art pre-trained 2.7 billion parameter BlenderBot model (Roller et al., 2020), which we fine-tune on our task.

Acting Score Model We can apply a retrieval model to also score the human's role-playing abilities. In this case, the context is the entire dialogue history, setting and the player's character persona as input to the encoder, while the candidates to score are the ones from the training set, as usual, plus additionally the human's (player's) actual response. The score given to the user is then proportional to the human response's rank amongst all the candidates ${ }^{2}$.

\subsection{Iterative Data Collection and Training}

After collecting a certain amount of episodes of conversational data between humans and models, one can consider using this data for training. We utilize the following observation: while the model utterances may contain many mistakes, it is assumed that a human sufficiently engaged provides high quality responses, even if the model responses are mistakes, and can thus be treated as gold, and used as a fully supervised signal. We thus separate the dialogue data into all possible (context, next utterance) pairs, and then only consider the pairs with human next utterances as training data. We also compare this to further filtering this set by scoring the quality of the human utterances, discarding those episodes (mini-games) with lower quality. We use the acting score model previously described for this purpose, summing the scores obtained across an episode, and discarding the episode if this value is less than $C$, where $C$ is a hyperparameter tuned on the validation set.

After training our model from a given round of collection, we can go back to the collection process utilizing instead the new model that has been trained on more data. The hypothesis is that the higher quality the model is: (i) the higher quality the human data will be as well; and (ii) the more likely the human players are to converse longer, increasing the data set size by larger amounts.

\subsection{Deployment-based Evaluation}

Apart from the collection-training cycle of our deployed lifelong learning setup, one can also in parallel perform evaluation. For each separate minigame (episode of dialogue) we can potentially deploy a different model for human-model conversation. We maintain a pool of models with differing architectures or hyperparameters, and select randomly from the pool in each episode. For any given episode we record whether the player continued playing to the next mini-game or not, which

\footnotetext{
${ }^{2}$ The player is awarded 2 stars if their response is in the top 2000, 3 stars in the top 1000, and 4 stars in the top 100 .
} 
we refer to as the continue rate. We can measure the quality of a model using its averaged continue rate over all players and episodes. In this way we can also perform model selection online.

\section{Experiments}

\subsection{Rounds of Learning}

We performed three rounds of our lifelong learning setup.

Round 1 consists of models trained on LIGHT MTurk data only. We train the retrieval model variants described in Section 4.1, and deploy them within the game.

Round 2 consists of models trained on LIGHT MTurk data $+50,982$ examples collected from the deployment of the Round 1 models, and again deploy these within the game.

Round 3 consists of models trained on LIGHT MTurk data $+50,982$ examples from Round 1 deployment + an additional 180,010 examples collected from Round 2 deployment.

\subsection{Data Collection}

While our setup is a lifelong learning setup and the models are still currently deployed and collecting data, for this paper we froze the collection at a given point in order to provide a data release and provide experimental results. The data statistics for the total newly collected dataset, called LIGHT WILD, over all rounds is shown in Table 1. Validation and test sets were extracted from a portion of the data ${ }^{3}$ from Round 2.

Table 2 compares this dataset to several existing commonly used open-domain dialogue datasets. The number of episodes and dialogue utterances is larger than many existing datasets, e.g. four times as many as LIGHT MTurk, and almost eight times that of Empathetic Dialog. Uniquely, our dataset contains human-model conversations, hence the total number of human utterances is actually half of the utterances, which is still twice as large as the number in LIGHT MTurk. Our dataset also has a large degree of diversity, which is important for tasks in general, and especially for open-domain dialogue. The number of unique locations and roles that can be played by speakers (characters) is large

\footnotetext{
${ }^{3}$ For validation and test we only use complete conversations, and where the player scored $\geq 9$ stars, to build higher quality evaluation sets.
}

(587 and 630, respectively). The number of players of the game at the time of freezing was over 13,000 , which also makes the diversity far larger than typical crowdsourced datasets, e.g. LIGHT MTurk involved 1,052 crowdworkers and Empathetic Dialog involved 810 crowdworkers. Finally, the number of unique tokens is larger in LIGHT WILD, indicating its diversity of language.

\subsection{Analysis of Results}

\subsubsection{Performance by Round}

While we only deployed retrieval models, we report experiments training both retrieval models and generative models on the data from the three rounds, selecting best hyperparameters using the validation set. We report the performance on three different test sets: LIGHT (MTurk) Seen and Unseen test sets (Urbanek et al., 2019), where unseen means that the test locations do not overlap with the training set locations, and our WILD test set. The results are given in Table 3. They show a steady increase in the Hits@1/20 metric (Top 1 accuracy given 19 random distractors) for the retrieval models over the rounds on all three test sets, and a similar decrease in perplexity (PPL) for the generative models. In particular there is a large jump in the performance on the WILD Test set between Rounds 1 and 2 as the training set switches from crowdsourced to in-distribution WILD data, and a further increase in Round 3 as more data is again collected and retrained on. While our WILD data is of a different distribution to the two LIGHT (MTurk) test sets, the data collection from our lifelong learning setup still gives gains on those tests as well. Our reported numbers, as far as we are aware, are the best reported numbers on these datasets, e.g. the original LIGHT paper reports $76.5 \%$ and $70.5 \%$ for the Seen and Unseen test sets, respectively (compared to our $87.72 \%$ and $83.48 \%$ ). Overall, we see clear gains from the extra data collected in our setup.

\subsubsection{Lifelong Learning Curves}

We construct learning curves given all the collected data to analyze the performance gain per new training example. We plot Hits@ 1/20 accuracy on the WILD validation set against the number of training examples, comparing data from WILD collection to LIGHT (Mturk). We also consider a 50/50 mix, where we equally sample from LIGHT MTurk and WILD to provide the next training example.

Figure 2 (left) shows the results. We observe that on a per-example basis our WILD data gives 


\begin{tabular}{lccccc}
\hline Data Type & Num. Epsiodes & Num. Utterances & Num. Human Utterances & Unique Locations & Unique Characters \\
\hline Training & 41,131 & 461,984 & 230,992 & 587 & 630 \\
Validation & 500 & 5,936 & 2,968 & 231 & 463 \\
Test & 1000 & 11,822 & 5,911 & 296 & 569 \\
\hline
\end{tabular}

Table 1: Data statistics of our lifelong learning deployment at the point where we froze collection for experiments reported within the paper and subsequent data release.

\begin{tabular}{lcccccc}
\hline Dataset & $\begin{array}{c}\text { Num. } \\
\text { Episodes }\end{array}$ & $\begin{array}{c}\text { Num. } \\
\text { Utterances }\end{array}$ & $\begin{array}{c}\text { Num. Human } \\
\text { Utterances }\end{array}$ & $\begin{array}{c}\text { Unique } \\
\text { Tokens }\end{array}$ & $\begin{array}{c}\text { Avg. Human } \\
\text { Utt. Length }\end{array}$ & $\begin{array}{c}\text { Number of } \\
\text { Humans }\end{array}$ \\
\hline PersonaChat (Zhang et al., 2018) & 8,939 & 131,438 & 131,438 & 18,688 & 11.9 & UNKNOWN \\
Wiz. of Wikipedia (Dinan et al., 2019b) & 18,430 & 166,787 & 166,787 & 52,490 & 19.7 & UNKNOWN \\
Empathetic Dialog (Rashkin et al., 2019) & 24,850 & 64,636 & 64,636 & 19,458 & 15.3 & 810 \\
Daily Dialog (Li et al., 2017) & 22,236 & 87,170 & 87,170 & 20,673 & 14.5 & UNKNown \\
LIGHT MTurk (Urbanek et al., 2019) & 8,538 & 110,877 & 110,877 & 33,789 & 18.3 & 1,052 \\
LIGHT WILD (this paper) & 41,131 & 461,984 & 230,992 & 47,526 & 11.9 & 13,188 \\
\hline
\end{tabular}

Table 2: Comparison of statistics of the dialogue data collected during deployment (bottom row) compared to several existing (mostly crowdsourced) datasets. Our data is around twice as large in terms of human utterances than these datasets, and $4 \mathrm{x}$ as large in terms of dialogue utterances (as our data consists of human-model conversations), while the cost to collect our data was only $1 / 5^{\text {th }}$ of the price per utterance of LIGHT MTurk, see Sec. 5.3.3.

more accuracy gains than LIGHT MTurk data, e.g. $83.59 \%$ for WILD compared to $80.63 \%$ for LIGHT, when limiting WILD to the same training set size as the total size of LIGHT. As the WILD dataset is more than twice as large this monotonically improves further, up to $85.95 \%$ using all of the WILD data. We observe that the improvements have not saturated and that further lifelong learning will bring further model improvements. Combining the two data sources, as shown in the LIGHT+WILD plot, brings yet further gains, up to $87.2 \%$. Overall, our collected WILD data has high quality as a learning signal for training models.

\subsubsection{Cost Learning Curves}

We plot similar learning curves, but as a function of the cost to collect the data instead of the number of training examples instead, see Figure 2 (right). Although we do not pay players to play the game, we did spend money to advertise the game online in order to attract players. We compare the cost per WILD example relatively to the cost per LIGHT (MTurk) example, where the $\mathrm{x}$-axis is scaled in units that are multiples of the cost required to achieve $80.63 \%$ using WILD data (as this is the performance of using all the LIGHT MTurk data together). We observe that it costs over $8 \mathrm{x}$ more to achieve the same accuracy using LIGHT (MTurk) data (see dashed horizontal line). The actual cost per utterance of WILD data is around $1 / 5^{\text {th }}$ of the price of LIGHT MTurk data ${ }^{4}$, but more than that, it is also relatively more effective per utterance in terms of metrics. For the same amount spent there is a large gap between the two systems, for example using all the WILD data gives a performance of $85.95 \%$, whereas for the same spend LIGHT MTurk only achieves $\sim 77.5 \%$. Overall, WILD deployment is relatively a very cost effective strategy.

\subsubsection{Deployment-based Evaluation}

Our lifelong learning setup deploys multiple models (see Sec. 4.3) at the same time randomly assigning them to concurrent users per episode (minigame). We can thus directly compare the quality of models via their continue rate.

Continue rates during Round 2 of collection comparing several model variants are given in Table 4. Continue rates are in the range of $68 \%-75 \%$, depending on the model, and we observe some clear trends. Most importantly, LIGHT+WILD trained models are superior to LIGHT only trained models, showing that our deployment/train cycle produces better models as judged by humans. Secondly, other factors in model design are important too, and our setup can effectively evaluate those. In particular, it was found that both our negative context training and decoding control enhancements (see Sec. 4.1) improve the continue rate, with both

\footnotetext{
${ }^{4}$ This is the cost of the $462 \mathrm{k}$ WILD utterances starting from a crowdsourced model base trained on 111k LIGHT utterances giving $573 \mathrm{k}$ utterances in total (see $\$ 5.1$ ), comparing to collecting an additional $462 \mathrm{k}$ examples via crowdsourcing.
} 


\begin{tabular}{lccc|ccc}
\hline & \multicolumn{3}{c|}{ Retrieval Model (Hits@ 1/20 $\Uparrow$ ) } & \multicolumn{3}{c}{ Generative Model (PPL $\Downarrow)$} \\
Model & LIGHT Test & LIGHT Test Unseen & WILD Test & LIGHT Test & LIGHT Test Unseen & WILD Test \\
\hline Round 1 & 87.12 & 82.43 & 81.61 & 12.67 & 11.81 & 13.42 \\
Round 2 & 87.65 & 82.70 & 84.60 & 12.57 & 11.74 & 12.31 \\
Round 3 & 87.72 & 83.48 & 87.63 & 12.54 & 11.75 & 11.79 \\
\hline
\end{tabular}

Table 3: Three rounds of training in our lifelong dialogue learning setup. Both retrieval and generative models trained on the data from the three rounds improve across both metrics on all three test sets.
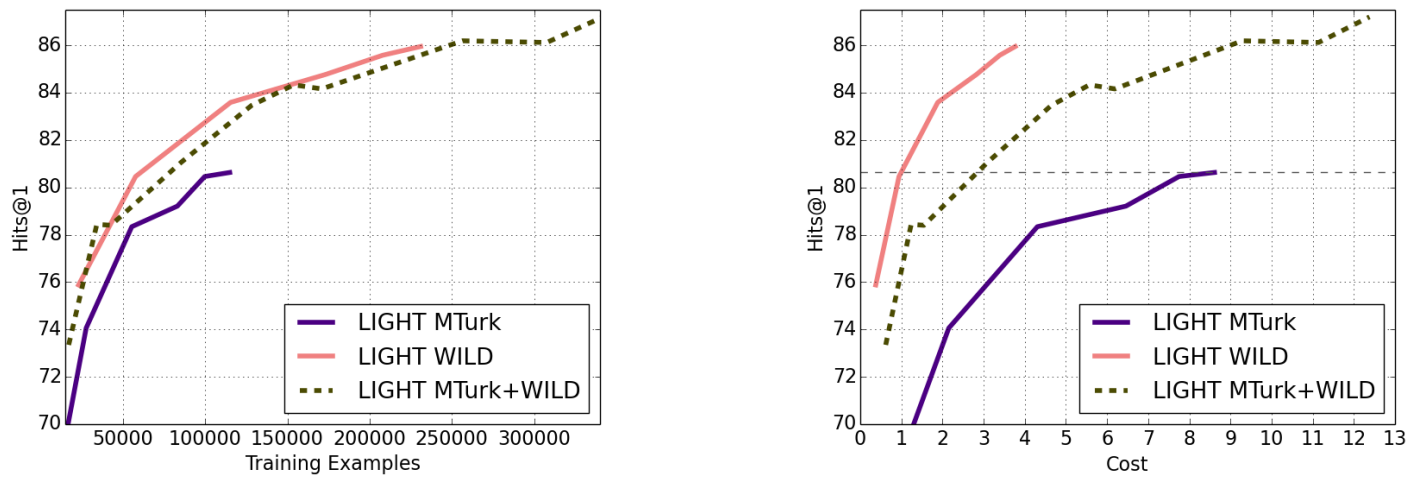

Figure 2: Hits@1/20 Accuracy on the LIGHT WILD validation set as a function of the number of training examples (left) or the cost of data collection (right). The cost axis is in units scaled by the cost of LIGHT WILD collection required to achieve the same performance as using the entire LIGHT MTurk dataset; it is more than $8 \times$ cheaper to use LIGHT WILD examples than LIGHT MTurk examples to achieve an accuracy of $80.63 \%$. We also show performance for models which equally sample data from LIGHT MTurk+WILD datasets for training; utilizing all the data from both sources yields the best performance. However, LIGHT WILD data gives better accuracy improvements per training example (left plot).

\begin{tabular}{lccc}
\hline Data & Neg.Ctxt & Dec.Ctrl & Continue Rate \\
\hline LIGHT & yes & no & $72.2 \pm 1.9 \%$ \\
LIGHT & yes & yes & $74.1 \pm 2.0 \%$ \\
LIGHT+WILD & yes & no & $73.6 \pm 1.8 \%$ \\
LIGHT+WILD & yes & yes & $75.2 \pm 2.0 \%$ \\
\hline
\end{tabular}

Table 4: Deployment-based Evaluation, comparing several metrics on data collected during Round 2 of collection for 90M PE models.

\begin{tabular}{lc}
\hline Model variation & $\Delta$ Continue Rate \\
\hline + WILD train data (Round 2) & $+1.3 \pm 0.7 \%$ \\
90M $\rightarrow$ 622M parameters PE & $-3.2 \pm 0.7 \%$ \\
+ Negative context training & $+2.6 \pm 1.3 \%$ \\
+ Decoding control & $+2.5 \pm 1.1 \%$ \\
\hline
\end{tabular}

Table 5: Deployment-based Evaluation: changes in continue rates for various model variants.

methods used together improving more. We confirm these conclusions in Table 5 where we show the change in continue rates when independently adjusting one of these factors, by averaging over model continue rates for other factors of variation.

We also observe the unexpected result that the larger models perform worse than the small models across the board on continue rates. Deeper analysis given in appendix D suggests that while the larger model makes less mistakes, it is more often seen as boring, which impacts a player's desire to continue playing. Understanding and controlling this tradeoff should be studied further.

\subsubsection{Data Quality}

Not every player is as engaged in the game as every other player, or produces as high quality dialogue. We hypothesize that we can predict which players produce higher quality data via the acting score model (Sec. 4.1), and that such higher quality data is relatively better for training models.

Figure 3 (right) shows the distribution over the WILD training set of predicted quality using the acting score model. We observe $83.7 \%$ of the episodes have a score above the minimum value of 6 (there are 6 turns, and on each turn a score between 14 is awarded, explaining the spike at the value of 6). Scores below 6 indicate incomplete dialogues, which only account for $4.0 \%$ of the data.

To assess whether these scores are indeed indicators of data quality, we selected an equal amount 

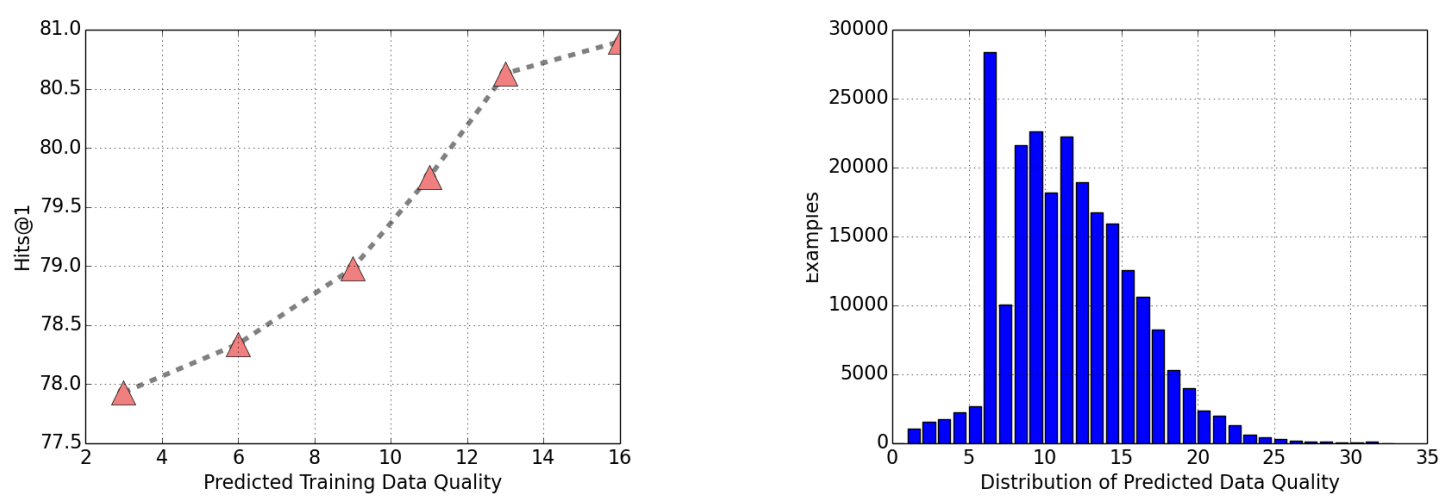

Figure 3: Predicted Data Quality. Left: Hits@1/20 accuracy on the WILD validation set when training with LIGHT MTurk + 10,000 examples from the WILD training set of a given predicted quality level, see Sec. 5.3.5. Data that is predicted to be higher quality yields improved validation accuracies. Right: The distribution of data quality predictions over the training set. A spike is seen at quality bin 6 because that is the lowest score one can achieve when completing a full episode ( 1 star per turn is awarded at minimum). Values lower than bin 5 indicate incomplete low-scoring episodes.

of 10,000 examples from each of the bins (1-5), (6), (7), .., (16) (grouping 1-5 together to make that group large enough) and compared them as training sources. We train a set of retrieval models on these training sources, where each model also has access to all of the LIGHT MTurk data (111k examples) in addition to the WILD 10k from their respective bins. The results are given in Figure 3 (left). We observe a monotonic improvement on the WILD validation set with increasing predicted quality. We see similar, but smaller gains on the LIGHT (Seen) validation set as well, e.g. $86.59 \%$ for quality bin 6 , and $87.11 \%$ for quality bin 16 .

While we can clearly select lower or higher quality data, we can also ask the question whether some of the data is so low quality we should simply remove it from the training data in order to get better performance. Experiments show that is not the case, and that even the lowest quality data does provide a useful signal, e.g. performance drops slightly from $87.06 \%$ to $86.69 \%$ on the WILD validation set if we remove bins lower than 6 , but otherwise training on all other data, and to $85.38 \%$ if we remove bins lower than 9 .

\subsubsection{Analysis of Data Distribution}

We can compare the dialogue data collected within our deployed system to crowdsourced data from LIGHT MTurk. We analyze over and underexpressed words in our dataset compared to the latter.

Calculating the top 70 most overexpressed words, all overexpressed at least $3.5 x$ relative to crowdsourced data, we note several interesting ob- servations about our data's distribution. There are more natural endings to conversations: e.g. "goodbye" $(4 \times)$ and "bye" $(4 \times)$ are overexpressed. There are overexpressed words associated with aggression: "stab" $(8.5 \times)$, "dagger" $(6.1 \times)$, "club" $(5.5 \times)$, "kills" $(4.9 \times)$, "blade" $(4.2 \times)$. There are overexpressed words associated with overtly friendly actions as well: "smiles" $(12.9 \times)$, "nods" $(10.9 \times)$, "kiss" $(6.1 \times)$, "hug" $(3.7 \times)$, and "bows" $(5.9 \times)$. There are more mentions of adventuring: "quest" $(5.4 \times)$, and other similar words not in the top 70 are overexpressed as well, such as "adventure" $(2.5 \times)$ and "mission" $(2.1 \times)$. There is an increased use of slang: "ur" $(93 \times)$, "u" $(28 \times)$, "yo" $(5 \times)$, "dude" $(6 \times)$. We note that some emojis exist as well that do not appear in the crowdsourced data.

In contrast, looking at the 70 most underexpressed words, all underexpressed by a factor of at least $1.3 \times$, we observed the following patterns. There are less mentions of village and farm life: "peasant", "fields" (both $2 \times$ underexpressed), "farm" and "crops" (both $1.9 \times)$, "harvest" $(1.8 \times)$, "villagers" $(1.7 \times)$, and "work" $(1.4 \times)$. There are less mention of passages of time: "week" $(2.1 \times)$, "year" $(1.9 \times)$, "days" $(1.8 \times)$.

Overall, we see a pattern that game players seek more exciting conversations, involving more emotional, action-packed interactions such as seeking quests, whereas crowdworkers are more evenkeeled, and discuss dry topics such as last year's harvest or taxes with more frequency. This is not unexpected as players often seek immediacy and larger-than-life experiences (Grodal et al., 2000). 


\section{Conclusion}

We have presented an approach for training dialogue agents during deployment with organic users utilizing a role-playing game between humans and bots. Detailed experiments show that one can collect high quality data that improves both automatic offline metrics and user engagement metrics. We find this exciting because this shows that it is possible to build continually improving models that learn from interacting with humans in the wild which represents a paradigm shift away from the limited static dataset setup that is prevalent in much of the work of the community. We make our code and data publicly available, and note our setup can be applied more generally, e.g., incorporating both dialogue and actions, or situated in other domains.

\section{References}

Andrew Carlson, Justin Betteridge, Bryan Kisiel, Burr Settles, Estevam R Hruschka, and Tom M Mitchell. 2010. Toward an architecture for never-ending language learning. In Twenty-Fourth AAAI conference on artificial intelligence.

Francisco M Castro, Manuel J Marín-Jiménez, Nicolás Guil, Cordelia Schmid, and Karteek Alahari. 2018. End-to-end incremental learning. In Proceedings of the European conference on computer vision (ECCV), pages 233-248.

Emily Dinan, Angela Fan, Adina Williams, Jack Urbanek, Douwe Kiela, and Jason Weston. 2020. Queens are powerful too: Mitigating gender bias in dialogue generation. In Proceedings of the 2020 Conference on Empirical Methods in Natural Language Processing (EMNLP), pages 8173-8188, Online. Association for Computational Linguistics.

Emily Dinan, Samuel Humeau, Bharath Chintagunta, and Jason Weston. 2019a. Build it break it fix it for dialogue safety: Robustness from adversarial human attack. In Proceedings of the 2019 Conference on Empirical Methods in Natural Language Processing and the 9th International Joint Conference on Natural Language Processing (EMNLP-IJCNLP), pages 4537-4546, Hong Kong, China. Association for Computational Linguistics.

Emily Dinan, Stephen Roller, Kurt Shuster, Angela Fan, Michael Auli, and Jason Weston. 2019b. Wizard of Wikipedia: Knowledge-powered conversational agents. In Proceedings of the International Conference on Learning Representations.

Torben Grodal et al. 2000. Video games and the pleasures of control. Media entertainment: The psychology of its appeal, pages 197-213.
Braden Hancock, Antoine Bordes, Pierre-Emmanuel Mazare, and Jason Weston. 2019. Learning from dialogue after deployment: Feed yourself, chatbot! In Proceedings of the 57th Annual Meeting of the Association for Computational Linguistics, pages 3667-3684, Florence, Italy. Association for Computational Linguistics.

Ari Holtzman, Jan Buys, Maxwell Forbes, and Yejin Choi. 2019. The curious case of neural text degeneration. In Proceedings of the International Conference on Learning Representations.

Matthew Horsfall and Andreas Oikonomou. 2011. A study of how different game play aspects can affect the popularity of role-playing video games. In 2011 16th International Conference on Computer Games (CGAMES), pages 63-69. IEEE.

Minlie Huang, Xiaoyan Zhu, and Jianfeng Gao. 2020. Challenges in building intelligent open-domain dia$\log$ systems. ACM Transactions on Information Systems (TOIS), 38(3):1-32.

Samuel Humeau, Kurt Shuster, Marie-Anne Lachaux, and Jason Weston. 2019. Poly-encoders: Architectures and pre-training strategies for fast and accurate multi-sentence scoring. In Proceedings of the International Conference on Learning Representations.

Esther Levin, Roberto Pieraccini, and Wieland Eckert. 2000. A stochastic model of human-machine interaction for learning dialog strategies. IEEE Transactions on speech and audio processing, 8(1):11-23.

Jiwei Li, Alexander H Miller, Sumit Chopra, Marc' Aurelio Ranzato, and Jason Weston. 2016a. Dialogue learning with human-in-the-loop. arXiv preprint arXiv:1611.09823.

Jiwei Li, Alexander H Miller, Sumit Chopra, Marc'Aurelio Ranzato, and Jason Weston. 2016b. Learning through dialogue interactions by asking questions. arXiv preprint arXiv:1612.04936.

Yanran Li, Hui Su, Xiaoyu Shen, Wenjie Li, Ziqiang Cao, and Shuzi Niu. 2017. DailyDialog: A manually labelled multi-turn dialogue dataset. In Proceedings of The 8th International Joint Conference on Natural Language Processing (IJCNLP 2017).

Bing Liu and Ian Lane. 2017. An end-to-end trainable neural network model with belief tracking for taskoriented dialog. In Proc. Interspeech 2017, pages 2506-2510.

Bing Liu, Gokhan Tür, Dilek Hakkani-Tür, Pararth Shah, and Larry Heck. 2018. Dialogue learning with human teaching and feedback in end-to-end trainable task-oriented dialogue systems. In Proceedings of the 2018 Conference of the North American Chapter of the Association for Computational Linguistics: Human Language Technologies, Volume 1 (Long Papers), pages 2060-2069, New Orleans, Louisiana. Association for Computational Linguistics. 
Sahisnu Mazumder, Bing Liu, Shuai Wang, and Nianzu Ma. 2019. Lifelong and interactive learning of factual knowledge in dialogues. In Proceedings of the 20th Annual SIGdial Meeting on Discourse and Dialogue, pages 21-31, Stockholm, Sweden. Association for Computational Linguistics.

Tom Mitchell, William Cohen, Estevam Hruschka, Partha Talukdar, Bo Yang, Justin Betteridge, Andrew Carlson, B Dalvi, Matt Gardner, Bryan Kisiel, et al. 2018. Never-ending learning. Communications of the ACM, 61(5):103-115.

Khanh Nguyen and Hal Daumé III. 2019. Help, anna! visual navigation with natural multimodal assistance via retrospective curiosity-encouraging imitation learning. In Proceedings of the 2019 Conference on Empirical Methods in Natural Language Processing and the 9th International Joint Conference on Natural Language Processing (EMNLPIJCNLP), pages 684-695, Hong Kong, China. Association for Computational Linguistics.

Aishwarya Padmakumar and Raymond J Mooney. 2020. Dialog as a vehicle for lifelong learning. arXiv preprint arXiv:2006.14767.

Ashwin Ram, Rohit Prasad, Chandra Khatri, Anu Venkatesh, Raefer Gabriel, Qing Liu, Jeff Nunn, Behnam Hedayatnia, Ming Cheng, Ashish Nagar, et al. 2018. Conversational ai: The science behind the alexa prize. arXiv preprint arXiv:1801.03604.

Hannah Rashkin, Eric Michael Smith, Margaret Li, and Y-Lan Boureau. 2019. Towards empathetic opendomain conversation models: A new benchmark and dataset. In Proceedings of the 57th Annual Meeting of the Association for Computational Linguistics, pages 5370-5381, Florence, Italy. Association for Computational Linguistics.

Verena Rieser and Oliver Lemon. 2011. Reinforcement learning for adaptive dialogue systems: a datadriven methodology for dialogue management and natural language generation. Springer Science \& Business Media.

Mark Bishop Ring. 1994. Continual learning in reinforcement environments. Ph.D. thesis, University of Texas at Austin Austin, Texas 78712.

Stephen Roller, Emily Dinan, Naman Goyal, Da Ju, Mary Williamson, Yinhan Liu, Jing Xu, Myle Ott, Kurt Shuster, Eric M Smith, et al. 2020. Recipes for building an open-domain chatbot. arXiv preprint arXiv:2004.13637.

Jost Schatzmann, Karl Weilhammer, Matt Stuttle, and Steve Young. 2006. A survey of statistical user simulation techniques for reinforcement-learning of dialogue management strategies. Knowledge Engineering Review, 21(2):97-126.

Abigail See, Stephen Roller, Douwe Kiela, and Jason Weston. 2019. What makes a good conversation? how controllable attributes affect human judgments.
In Proceedings of the 2019 Conference of the North American Chapter of the Association for Computational Linguistics, pages 1702-1723. ACL.

Iulian V Serban, Chinnadhurai Sankar, Mathieu Germain, Saizheng Zhang, Zhouhan Lin, Sandeep Subramanian, Taesup Kim, Michael Pieper, Sarath Chandar, Nan Rosemary Ke, et al. 2017. A deep reinforcement learning chatbot. arXiv preprint arXiv:1709.02349.

Daniel L Silver, Qiang Yang, and Lianghao Li. 2013. Lifelong machine learning systems: Beyond learning algorithms. In 2013 AAAI spring symposium series.

Jack Urbanek, Angela Fan, Siddharth Karamcheti, Saachi Jain, Samuel Humeau, Emily Dinan, Tim Rocktäschel, Douwe Kiela, Arthur Szlam, and Jason Weston. 2019. Learning to speak and act in a fantasy text adventure game. In Proceedings of the 2019 Conference on Empirical Methods in Natural Language Processing and the 9th International Joint Conference on Natural Language Processing (EMNLP-IJCNLP), pages 673-683, Hong Kong, China. Association for Computational Linguistics.

Luis Von Ahn. 2006. Games with a purpose. Computer, 39(6):92-94.

Harm de Vries, Dzmitry Bahdanau, and Christopher Manning. 2020. Towards ecologically valid research on language user interfaces. arXiv preprint arXiv:2007.14435.

Sean Welleck, Ilia Kulikov, Stephen Roller, Emily Dinan, Kyunghyun Cho, and Jason Weston. 2020. Neural text generation with unlikelihood training. In International Conference on Learning Representations.

Saizheng Zhang, Emily Dinan, Jack Urbanek, Arthur Szlam, Douwe Kiela, and Jason Weston. 2018. Personalizing dialogue agents: I have a dog, do you have pets too? In Proceedings of the 56th Annual Meeting of the Association for Computational Linguistics, pages 2204-2213. ACL. 


\section{A Further Game Details}

Other Gamification Steps The acting scores (between 1-5 stars per turn) are accumulated, and a player's total score is presented on a leaderboard compared to all other players, providing further motivation to reach the top of the leaderboard. We also award "badges" if, for a given dialogue, a certain number of points is collected (11 for 1 badge, 16 for 2): the badges represent the characters in the game, motivating the desire to role-play all the characters in the game, and collect all the badges.

License Agreement and Public Release Upon entry to the game, players are asked to agree to the use and release of the resulting game data as a publicly available dataset for research purposes. They are urged to stick to their assigned characters in the game, and hence should not use any personally identifying information, which the terms also tell them explicitly not to share. In the released data, no other information about the player is retained except for the messages they send in the game.

Game Safety We employ a safety classifier (Dinan et al., 2019a) on both human and model turns. For safety reasons, we limit our deployed dialogue models to be retrieval models, so that we could vet the entire set of candidates for offensive language before run-time (we evaluate generative models in an offline setting only). The set of settings and character personas were all also vetted for offensive language. Additionally, gender bias concerns have been previously studied within the available LIGHT MTurk training set (Dinan et al., 2020), and we make use of that publicly available data here as well. We note that, compared to other deployed dialogue systems, there is an extra level of indirection due to playing characters in a game that makes language relatively less offensive. For example, a thief in the game saying "I'm going to steal your money" to another game character is far less offensive compared to a digital assistant saying it directly to a human user.

\section{B Game Screenshots}

We show game screenshots in Figures 5 and 6.

\section{Using WILD Model responses}

In our main results we use the human utterances collected from the role-playing game to form the WILD dataset targets, the hypothesis being that model utterances may or not be correct, and so are not as good a signal. A contrasting view could see training on the model utterance data as a kind of distillation of the previous model's knowledge. To test the performance of WILD human vs. model utterances, we conducted further experiments comparing them to each other. We observe a significant drop in Hits@ 1/20 performance using the model utterances for training on the WILD validation set (86.05\% for human utterances, and $73.96 \%$ for model), and similarly on the LIGHT validation set ( $82.32 \%$ for human, and $77.56 \%$ for model).

A similar trend emerges when evaluating our generative models. As before, we see a drop in performance when training on WILD model utterances vs. training on WILD human utterances, manifesting as significant increases in perplexity on the WILD validation set (12.05 for human utterances, and 15.15 for model) as well as the LIGHT validation set (13.71 for human utterances, and 14.76 for model).

With the generative models, we additionally explored multi-task training with both the model and human utterances. In this regime, we introduced a special control token to indicate whether the desired response was produced by a model or a human; at inference time, we always provide the "human" control token. Including the control tokens in the training setup yields lower perplexities compared to vanilla multi-task training on both the WILD validation set (12.10 with control, 12.34 without) and the LIGHT validation set (13.60 with, 13.65 without). We note that, overall, multi-task training yields marginally worse performance on the WILD validation set and marginally better performance on the LIGHT validation set (see numbers in previous paragraph).

\section{Comparing Small and Large Model Variants}

We evaluated the differences between the small and large retrieval models (Sec. 4.1) during deployment to analyze the differences between them. Results for both large and small models are shown in Table 7. The expectation tends to be that larger models would perform better, especially when the automatic metrics reflected this case. To eliminate the possibility that difficulty in tuning the decoding control differed between the small and large models, we launched a task to crowdworkers to evaluate the models shown in rows 3 and 8 of Table 4 . These 


\begin{tabular}{l|cccc|cc}
\hline Model & Contradiction & Mistaken Identity or Location & Off-topic & Repetitive or Boring & Rating & Count \\
\hline 90M PE & $3.9 \%$ & $9.1 \%$ & $8.4 \%$ & $2.1 \%$ & 3.67 & 88 \\
$622 \mathrm{M} \mathrm{PE}$ & $3.0 \%$ & $5.3 \%$ & $6.0 \%$ & $5.3 \%$ & 3.69 & 91 \\
\hline
\end{tabular}

Table 6: Percentage of utterances flagged with an issue alongside overall satisfaction, by model.

correspond to the small and large models trained with LIGHT + WILD data without decoding control, further noted as LW90M and LW622M.

Workers were asked to have a 12-turn conversation with a model (6 turns each), and evaluate each model utterance with respect to some of the most common mistakes we observed models making. They were then asked to provide an overall score for the model, answering "How much fun did you have Role-playing with your chat partner? Would you have a similar conversation with this chat partner in the future?". Results are given in Table 6 , listing the percentage of utterances falling into each mistake type.

We observed that LW622M avoided a number of common mistakes when compared to LW90M, contradicting itself less frequently, assuming the role of the wrong character or using a wrong location less frequently, and going off-topic less frequently. These types of mistakes were common complaints of players interacting with our game, and all seemed to be related to having somewhat bad experiences. LW622M also had a higher percentage of conversations without any listed issues, with $34.1 \%$ compared to $27.3 \%$ for LW90M.

LW622M however was rated as being repetitive or boring more than twice as frequently as the smaller model. This difference could be supported in that LW622M used utterances that were on average 2 words shorter than those of LW90M over these evaluations. This issue could explain the phenomenon where players are more likely to leave after interacting with a larger model than a smaller one. The only thing really encouraging unpaid players to continue interacting with our models is that they are fun and engaging, and while it is possible to overlook the model making a mistake if it is still somewhat fun, it is likely less possible to remain engaged when the model is actually boring and repetitive.

While LW622M is a better model across several aspects, it is clear from our live deployment evaluations that something is lost in scaling up from LW90M. Comparing these models with real players lets us see this issue, and moving forward should lead us to search for a model that does not become more boring when learning to not make mistakes.

\begin{tabular}{lcccc}
\hline Model & Train Data & $\begin{array}{c}\text { Negative } \\
\text { Context }\end{array}$ & $\begin{array}{c}\text { Decoding } \\
\text { Control }\end{array}$ & $\begin{array}{c}\text { Continuation } \\
\text { Rate }\end{array}$ \\
\hline 90M PE & LIGHT & yes & no & $72.2 \pm 1.9 \%$ \\
90M PE & LIGHT & yes & yes & $74.1 \pm 2.0 \%$ \\
90M PE & LIGHT + WILD & yes & no & $73.6 \pm 1.8 \%$ \\
90M PE & LIGHT + WILD & yes & yes & $75.2 \pm 2.0 \%$ \\
\hline \multirow{2}{*}{ 622M PE } & LIGHT & no & no & $68.2 \pm 1.4 \%$ \\
622M PE & LIGHT & yes & no & $69.9 \pm 1.9 \%$ \\
622M PE & LIGHT & yes & yes & $69.9 \pm 2.0 \%$ \\
622M PE & LIGHT + WILD & yes & no & $70.6 \pm 2.1 \%$ \\
622M PE & LIGHT + WILD & yes & yes & $71.8 \pm 1.9 \%$ \\
\hline
\end{tabular}

Table 7: Deployment-based Evaluation, comparing several metrics on data collected during round 2 .

\section{E Observations on Gamification}

Just as the design of a crowdsourcing task will affect the cost and quality of data, this is likely even more the case in the design of a game. If the design is poor, players will not play it at all; whereas in contrast to paying crowdworkers, if players really like a game, they are willing to pay to play it. Accordingly, the plots we presented in Figure 2 represent the results of our particular game design; there may well be a design with vastly more cost efficient learning rates. While a full of study of the elements of game design is outside of the scope of this paper, we note that for adjustments we did make to the game after initial deployment we observed large changes in user behavior. For example, after the addition of the three user controls for how to continue the game loop after an episode is finished (as described in Sec. 3), compared to only a single choice, we saw an increase in the continue rate by $3.3 \pm 1.6 \%$ when using the same model.

Model quality also affects cost and quality of the data collected. Noting the effects of changing gamification options (alongside other hard-totrack circumstances) we only report continue rate comparisons between models relative to runs in the same batch. Still, players' enjoyment of these models (as estimated by continue rate in Table 4) directly changes how much they engage with the game. As such it is more expensive to test models that are worse for the game experience (which we would consider fair from a player perspective). Hence, as models improve, costs actually go down, enabling to collect data at higher rate. 


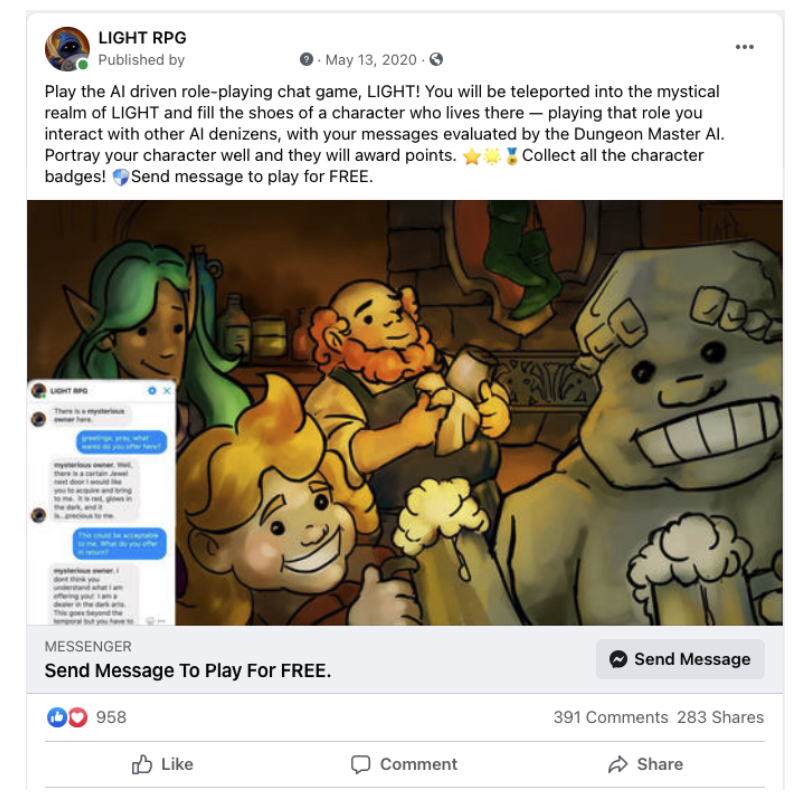

Figure 4: Example advertisement used to draw interest to the deployed LIGHT chat game

\section{$0 y$}

545145 Welcome back to the world of LIGHT! 745

The dungeon master $\%$ is glad to see you again Tunatete!

808

2 Click PLAY below to start playing! Click OPTIONS below for more options.

OPTIONS:

1. INSTRUCTIONS : view the instructions again.

2. CHANGE USERNAME : change your display name for the leaderboard.

3. LEADERBOARD : view the leaderboard.

4. CHARACTERS : view collected character badges.

5. POLICIES : reread our policies.

Game: Your rank is 461 out of 13119.

Your total score is 160 .

LEADERBOARD:

1. Yuhhhhhhhh: 7452

2. GoodKnight: 1488

3. TheDungeonMaster: 1381

4. Verity: 1348

5. Qerrathien: 1311

- Click PLAY below to start playing! Click OPTIONS below for more options.

Here are all of the badges the Dungeon Master has awarded you for exemplary performance so far.

You've collected 6 badges: o hill Aisile

(9) Click PLAY below to start playing! Click OPTIONS below for more options.

Figure 5: Screenshots of the instructions of the LIGHT role-playing game. 
You are in the Large cliffs.

Towering cliffs of gray granite line the coast. A few meters of sand lie between

the ocean and the base of the cliffs.

A mountain lion is here.

You are carrying a dustpan and a hand plow.

2 mountain lion. What are you doing here goat?

: $:(3$ Star!!)

mountain lion. Must be hard to find this far up the mountain?

WฟW (3 Star!!)

mountain lion. My master is visiting the maidens cottage and I am able to exercise in the meadow in the mean time. mountain lion. He is a noble man. You shouldnt ask questions about my master. mountain lion. Lots of cardio, squats, and bench presses! I may be a servant, but I have a lot of time to workout!

\section{(3 Star!!)}

mountain lion. Ach, well, its been many a year since lve been called strong. Although I was once a fierce warrior in my day!

Swish! A portal door opens in the thin air. The Dungeon Master strides out and turns to you. Good work! The Dungeon Master gives you a badge to celebrate your role-playing skills: "Y You already had one of these, but the more the merrier I suppose.

You've collected 6 badges so far.

(2) Your score increased by 12 points from your role-playing skills!

Figure 6: Gameplay Screenshots of the LIGHT role-playing game. 